\title{
Chromonychia Secondary to Chemotherapy
}

\author{
Marien Lopes Clarice Jordão Rachel Grynszpan Celso Sodré \\ Marcia Ramos-e-Silva
}

Sector of Dermatology and Post-Graduation Course in Dermatology, University Hospital and School of Medicine, Federal University of Rio de Janeiro, Rio de Janeiro, Brazil

\section{Key Words}

Chemotherapy · Chromonychia $\cdot$ Cyclophosphamide $\cdot$ Doxorubicin $\cdot$ Hydroxyurea

\begin{abstract}
Chemotherapy drugs can affect the skin and its appendages. Several clinical presentations can be observed, depending on the affected structure. The most common dermatological side effect is chromonychia. The main causative agents are: (1) cyclophosphamide, which can provoke a diffuse, black pigmentation, longitudinal striae and dark grey pigmentation located proximally on the nails; (2) doxorubicin, which promotes dark brown bands alternating with white striae and dark brown pigmentation in transverse bands, and (3) hydroxyurea, which produces a distal, diffuse, dark brown pigmentation. In the majority of cases, the effects are reversible after the suspension of the causative agent for a few months. We report a patient who developed chromonychia while undergoing treatment with cyclophosphamide, vincristine, doxorubicin, dexamethasone, methotrexate and cytarabine for acute lymphocytic leukemia.
\end{abstract}

\section{Introduction}

The number of patients undergoing chemotherapy grows year by year. Despite their efficacy in controlling the cancer, chemotherapy drugs are not inert, and they cause damage to the human body. The skin and its extensions can be affected, especially hair and nails.

Regarding nail involvement, diverse clinical features can be observed, according to the structure affected and the severity of the damage caused [1]. The most common chemotherapeutic agents implicated in nail changes are cyclophosphamide, doxorubicin and hydroxyurea [2, 3]. In the majority of cases, the effects are fully reversible after a few months once the causative agent has been withdrawn $[2,4]$. 


\section{Case Report}

A 25-year-old male patient was admitted to the hospital's hematology ward with the diagnosis of acute lymphocytic leukemia. He was being treated with the hyper-CVAD chemotherapy regimen. The hyper-CVAD regimen delivers a combination of drugs in 2 cycles (cyclophosphamide, vincristine, doxorubicin and dexamethasone in cycle A, methotrexate and cytarabine in cycle B), split into smaller doses and administered at more frequent intervals in order to minimize side effects.

During hospitalization, a dermatology assessment was requested. The dermatological examination showed nail injury characterized by transverse, brown-gray hyperpigmentation affecting the proximal half of the nail plate, with a brown band delimiting the healthy nail plate on all fingers (fig. 1, fig. 2, fig. 3).

\section{Discussion}

Chromonychia induced by chemotherapeutic agents has different forms, the most common being melanonychia $[2,4,5]$. The dark pigmentation in nails can be diffuse, transverse or longitudinal and can coexist with skin and mucosal pigmentation $[2,4,6]$.

It has been suggested that chemotherapy drug regimens influence the incidence of changes that may result from toxicity in the nail matrix, nail bed, periungual tissue and digits' vessels [2,6]. Although several chemotherapeutic agents can cause these changes, those most associated with these side effects are cyclophosphamide, doxorubicin and hydroxyurea [2, 3].

Cyclophosphamide is an alkylating agent whose main target is the cell cycle; thus, tissues with high proliferation rates are more susceptible. Alkylating agents form cross-links with DNA filaments and prevent DNA replication, therefore entailing cytotoxicity. Cyclophosphamide can cause nail changes such as diffuse, black pigmentation, longitudinal striae ranging in color from slate gray to black, and diffuse, dark gray pigmentation located proximally, with overlying transverse, black bands $[2,4]$. This drug-induced nail pigmentation starts from the proximal edge of the nail and proceeds towards the distal edge [2]. Upon withdrawal of the drug, regression occurs in a similar fashion, starting at the proximal edge. It takes approximately 4-6 months [2, 3]. In $60 \%$ of patients, cyclophosphamide is also associated with pigmentation of the oral mucosa and tongue, which also regresses upon withdrawal of the drug $[2,4]$.

Doxorubicin is an antiblastic antibiotic which prevents the proliferation and functioning of tumor cells. Its side effects include skin and nail hyperpigmentation, alopecia, skin rash, pruritus, photosensitivity, urticaria, acral erythema and palmoplantar erythrodysesthesia. Nail changes such as transverse, dark brown bands alternating with white stripes [2, 7] and dark brown diffuse pigmentation bands 4-5 mm wide, which affect two-thirds of the distal portion of the nail, also occur [2,5]. Additionally, doxorubicin can cause subungual keratosis, onycholysis and thickening of the nail plate [2].

Hydroxyurea causes an immediate inhibition of DNA synthesis by acting as an inhibitor of ribonucleotide reductase, without interfering in the synthesis of ribonucleic acid or protein. It triggers a more distal nail pigmentation than cyclophosphamide. This pigmentation has a diffuse, dark brown color which may appear as single or double transverse bands $[2,4]$. 
Lopes et al.: Chromonychia Secondary to Chemotherapy

\section{Conclusion}

Ungual hyperpigmentation induced by chemotherapy is not uncommon, although its occurrence is underestimated and seldom reported, especially in patients with dark skin phototypes, such as our patient. In this case, the drugs most probably implicated are doxorubicin and cyclophosphamide because they produce ungual alterations very similar to the ones seen in our patient; his chemotherapeutic scheme included both of them.

\section{Disclosure Statement}

The authors have no conflict of interest related to this publication.

\section{References}

1 Piraccini BM, Iorizzo M: Drug reactions affecting the nail unit: diagnosis and management. Dermatol Clin 2007;25:215-221.

-2 Ranawaka RR: Patterns of chromonychia during chemotherapy in patients with skin type $V$ and outcome after 1 year of follow-up. Clin Exp Dermatol 2009;34:e920-e926.

-3 Piraccini BM, Tosti A: Drug-induced nail disorders: incidence, management and prognosis. Drug Saf 1999;21:187-201.

4 Gupta A, Parakh A, Dubey AP: Chemotherapy induced nail changes. Indian J Dermatol 2008;53:204-205.

5 Gilbar P, Hain A, Peereboom VM: Nail toxicity induced by cancer chemotherapy. J Oncol Pharm Pract 2009;15:143-155.

6 Dasanu CA, Vaillant JG, Alexandrescu DT: Distinct patterns of chromonychia, Beau's lines, and melanoderma seen with vincristine, adriamycin, dexamethasone therapy for multiple myeloma. Dermatol Online J 2006;12:10.

7 Unamuno P, Fernández-López E, Santos C: Leukonychia due to cytostatic agents. Clin Exp Dermatol 1992;17:273-274. 


\section{Case Reports in Dermatology}

\begin{tabular}{l|l}
\hline Case Rep Dermatol 2013;5:163-167 \\
\hline DOI: 10.1159/000351874 & $\begin{array}{l}\text { C 2013 S. Karger AG, Basel } \\
\text { www.karger.com/cde }\end{array}$ \\
\hline
\end{tabular}

Lopes et al.: Chromonychia Secondary to Chemotherapy

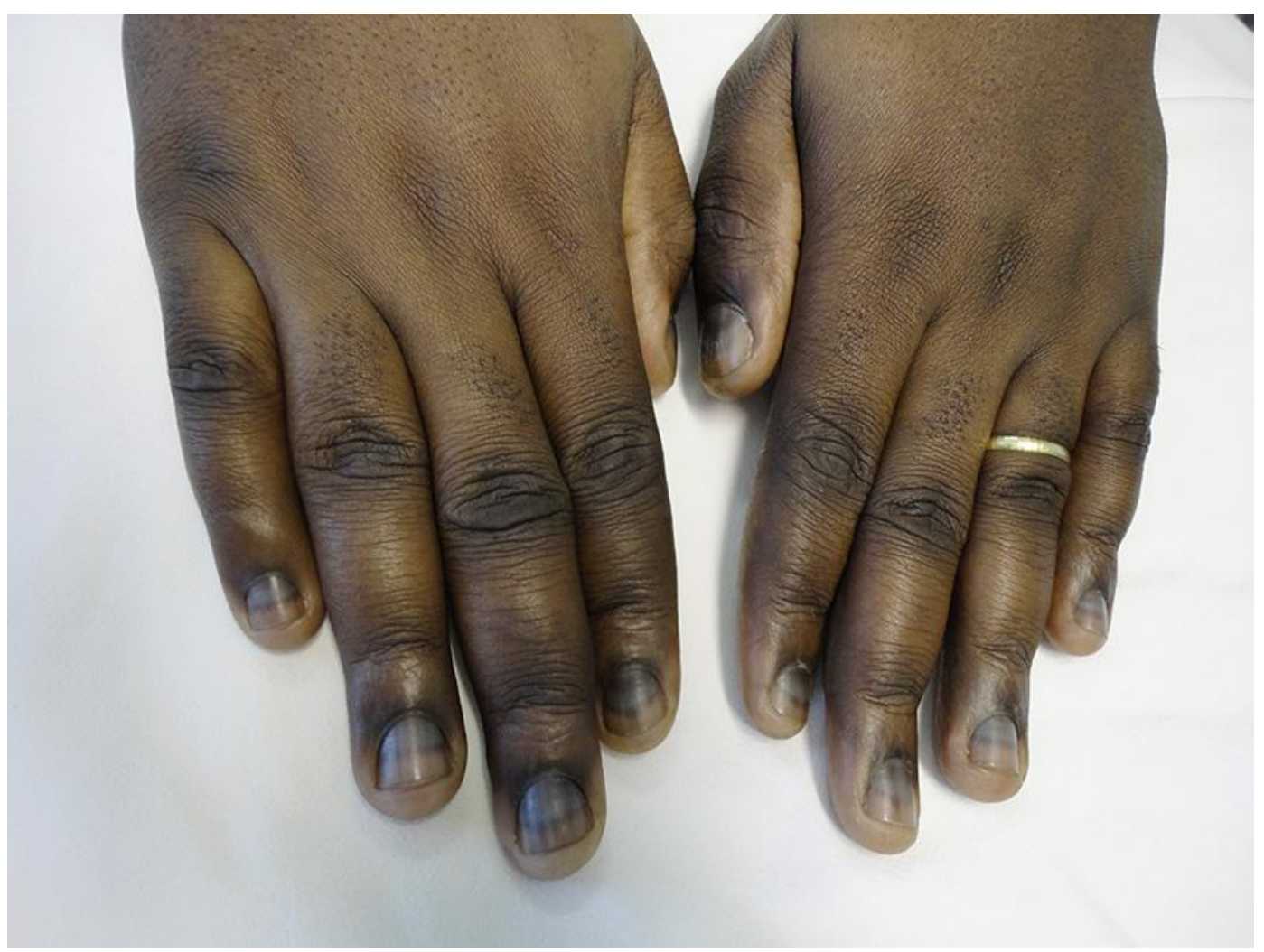

Fig. 1. Nail involvement of all fingers.

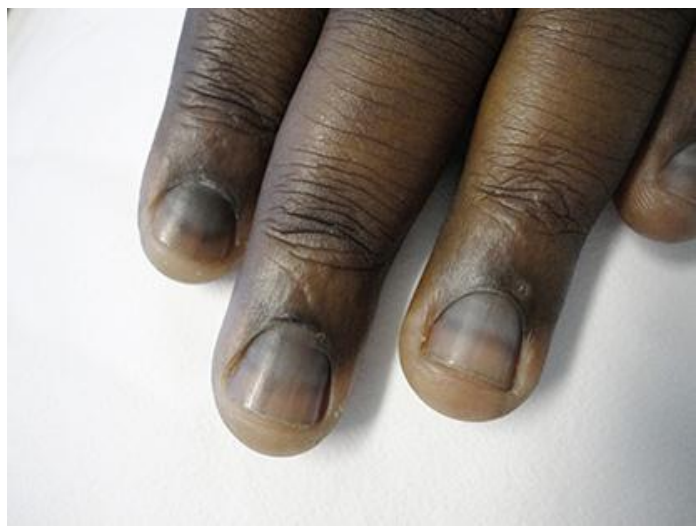

Fig. 2. Transverse, brown-greyish hyperpigmentation on the proximal half of the nail lamina, with a brownish band delimiting the healthy nail. 


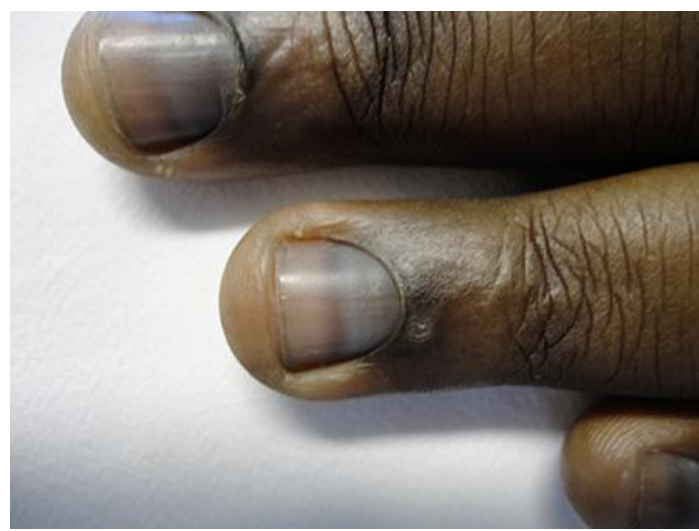

Fig. 3. Close-up of the lesion. 